\title{
SUSPENSÃO DO ROCIADO NO COMBATE AO TRIATOMA INFESTANS EM ÁREAS DO ESTADO DE SÃO PAULO, BRASIL
}

\author{
Eduardo Olavo da ROCHA e SILVA (1) \\ Jairo DIAS JÚNIOR (1) \\ Odilon Ferreira GUARITA (1)
}

\begin{abstract}
Rocha e Silva, E. O. da; Dias Júnior, J. \& Guarita, O. F. - Suspensão do rociado no combate ao Triatoma infestans em áreas do Estado de São Paulo, Brasil. Rev. Saúde públ., S. Paulo, 3(2):173-181, dez. 1969.

RESumo - Foi relatada a evolução do combate ao $T$. infestans, acentuando-se que inicialmente ocorreu uma queda na densidade de exemplares nos municipios periòdicamente trabalhados. Esta foi acompanhada da queda dos índices de infecção pelo $T$. cruzi e das reações de Machado Guerreiro positivas. Quando as condições se tornaram adequadas, introduziu-se o método seletivo de Pedreira de Freitas, o qual possibilitou a melhoria das técnicas de borrifação e captura e ainda a ampliação da área protegida. Considerou-se que uma campanha de contrôle dos vetores domiciliários da doença de Chagas, apresenta distintas etapas. Procurou-se esclarecer quando e como, em São Paulo, conseguiu-se, em alguns municípios, chegar à fase de Vigilância, preconizada por Emmanuel Dias. Estabeleceu-se uma área pilôto, onde estão sendo estudados métodos de trabalho e adestrado o pessoal lotado nas áreas em Vigilância. Procurou-se mostrar que o $T$. sordida e o $P$. megistus não vêm respondendo satisfatòriamente aos atuais métodos de contrôle.
\end{abstract}

\section{I N T R O D U C $\mathrm{AO}$}

Iniciadas as atividades de contrôle da endemia chagásica, em maio de 1959 , pelo então Serviço de Profilaxia da Malária (SPM) já em dezembro do ano seguinte, Lima \& Silva ${ }^{8}$ (1952) assinalavam que dos 369 municípios então existentes, 231 apresentavam-se infestados por triatomínios. A infestação das moradias era causada principalmente pela presença do Triatoma infestans, cujo índice geral de infecção pelo Trypanosoma (Schizotrypanum) cruzi, em 12.668 exemplares exa- minados, atingia $11,92 \%$ - CorrêA, LIma \& CaRvalho $^{3}$ (1952). Na mesma época, UnTI \& Silva ${ }^{15}$ (1952) mostraram, através de reações de fixação de complemento, realizadas entre moradores de 65 municipios infestados, que o percentual de positividade alcançava $20,02 \%$.

Reconhecido o efeito letal do hexaclorociclohexano (BHC) sôbre os triatomíneos, em ensaios de campo realizados por Dias \& Pellegrino ${ }^{6}$ (1948), foi êle escolhido para ser aspergido nas casas $\mathrm{e}$

Recebido para publicação em 15-8-1969.

(1) Da Divisão de Combate a Vetores da Secretaria da Saúde Pública do Estado de São Paulo - São Paulo, Brasil. 
ROCHA e SILVA, E. O. da; DIAS JR., J. \& GUARITA, O. F. - Suspensão do rociato no combate ao Triatoma infestans. Rev. Saúde públ., S. Paulo, 3(2):173-181, dez. 1969.

anexos da área infestada do Estado, sob a forma de pó molhável, inicialmente na dose de $0,25 \mathrm{~g}$ e posteriormente de $0,50 \mathrm{~g}$ de material ativo por $\mathrm{m}^{2}$ - Schiavi; Lima \& RAMOS $^{14}$ (1952).

Ainda era elevado, em fevereiro de 1958, 0 índice geral de infecção do $T$. infestans, pois CorrêA \& FERREIRA ${ }^{1}$ (1959) publicaram uma relação das espécies de triatomíneos capturados até aquela data, no Estado de São Paulo, onde 9,37\% dos exemplares da referida espécie se apresentavam infectados pelos $T$. cruzi, em um total de 156.920 examinados.

O emprêgo, até os primeiros anos da década de sessenta, de uma técnica de borrifação, sob certos aspectos ainda precária, compensada porém pela continuidade com que foram trabalhados alguns municípios e pelo hábito domiciliário ou peridomiciliário do $T$. infestans, foi $o$ que ocasionou, supõe-se, a queda de sua densidade em algumas áreas anualmente trabalhadas (primeira etapa do contrôle). Essa queda na densidade dos vetores domiciliários trouxe como conseqüência a re. dução da transmissão da doença ao homem. Coutinho ${ }^{4}$ (1962), autorizado pelo então Serviço de Erradicação da Malária e Profilaxia da Doença de Chagas (SEMPDC), publicou os resultados de um inquérito sorológico levado a efeito por êsse Serviço na zona rural, onde, de um total de 15.271 reações de Ma- chado Guerreiro, $9,31 \%$ se apresentavam positivas. No grupo etário de 0 a 9 anos a positividade alcançou $6,33 \%$ em 3.365 amostras. $O$ mesmo fato pode ser indiretamente observado pela leitura da Tabela 1 e Figura 1, que mostra a queda dos índices de infecção dos $T$. infestans capturados entre 1951 e 1968.

Após o término da fase de Ataque (1964), em grande parte da área malárica do Estado de São Paulo, as atividades de contrôle dos vetores da doença de Chagas receberam forte impulso. A experiência adquirida pelos técnicos do SEMPDC em operaçôes de campo (rociado e epidemiologia), cuidadosamente executadas e supervisionadas e a utilização das técnicas dessa campanha de erradicação, devidamente adaptadas às condições peculiares da epidemiologia e profilaxia da doença de Chagas, possibilitaram-nos, em poucos anos, intensificar a luta contra os triatomíneos vetores. Os trabalhos, a partir dessa época, foram executados de tal maneira que, uma vez reduzida a densidade de triatomíneos nos focos, conseguiu-se em seguida, a extinção de um grande número dêles (segunda etapa do contrôle) - Rocha e Silva \& FRANÇA ${ }^{12}(1965 / 6)$.

A técnica de expurgo, já então aprimorada, e a melhoria da pesquisa e captura dos exemplares encontrados nas moradias, permitiram ao SEMPDC, a par-

T A B E L A I

Movimento geral - Triatoma infestans

Periodo: 1951-1968

\begin{tabular}{c|c|c|c|c|c}
\hline \multirow{2}{*}{ Período } & \multicolumn{3}{|c|}{ Número de Exemplares } & \multicolumn{2}{c}{$\%$ de } \\
\cline { 2 - 5 } & Capturados & Examinados & Infectados & Exam. & Infec. \\
\hline 1951 a 1955 & 203.058 & 118.931 & 10.825 & 58,57 & 9,10 \\
1956 a 1960 & 86.198 & 56.373 & 4.589 & 65,39 & 8,14 \\
1961 a 1965 & 108.322 & 72.072 & 2.441 & 66,53 & 3,38 \\
1966 a 1968 & 165.970 & 132.924 & 3.686 & 80,00 & 2,79 \\
\hline
\end{tabular}




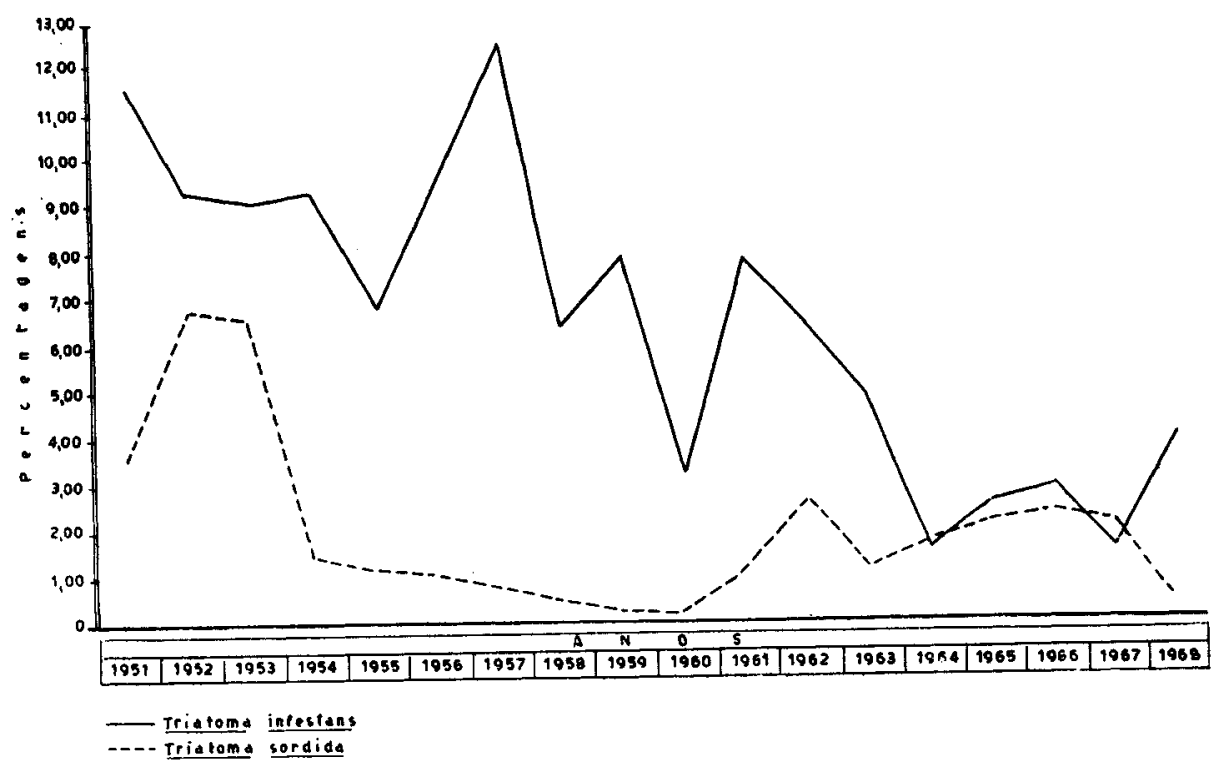

Fig. 1 - Indices de infeccão do T. infestans e $T$. sordida pelo Trypanosoma cruzi, no Estado de São Paulo, Brasil.

tir do segundo semestre de 1967, introduzir o método seletivo preconizado por Pedreira de Freitas ${ }^{10}$ (1963). A utilização dêsse método, nessa ocasião, baseou-se inclusive em justificativa econômica, pela determinação da infestação de equilíbrio $\left(\mathrm{X}_{\mathrm{e}}\right)$, segundo fórmula concebida por Reis; França \& Rocha e SilvA ${ }^{11}$ (1969).

0 rendimento do trabalho e a econo. mia proporcionada pelo emprêgo do método seletivo, possibilitaram a ampliação da área protegida, assim como o uso de técnicas de combate aos focos, cada vez mais eficientes. Surgiram, em decorrência, o uso conjunto de inseticida (BHC a $30 \%$ ) e desalojante (Piriza-0540 - emulsão) (Paulini; Fomm \& Guedes ${ }^{9}, 1961$ e CorrêA; Fomm \& Dias Jr. $\left.{ }^{2}, 1967\right)$; a revisão das casas infestadas 60 a 90 dias após o primeiro expurgo e ainda a pesquisa manual com laterna e desalojante.

Em 1968, foram pesquisadas 885.992 casas (18.626 infestadas), o que corresponde à proteção dada a cêrca de ....
4.500.000 habitantes da zona rural distribuídas entre 422 municípios, dos quais 182 infestados pelo $T$. infestans e dêstes, 77 com triatomineos infectados. Apenas 2,1 das casas pesquisadas se apresentaram infestadas, não se levando em conta neste dado, a espécie de triatomíneo encontrada, nem se a casa estava ou não habitada. Foram capturados nas casas positivas para triatomíneos, 44.055 exemplares, proporcionado uma média relativa de menos de 3 exemplares por casa infestada. Dêsses triatomíneos, 1.871 exemplares foram considerados, após exame de laboratório, infectados por tripanossomas do tipo cruzi ou seja $4,6 \%$ do total dos capturados no interior dos domicílios. Número êsse elevado ainda, sem dúvida, por refletir a desigualdade até há pouco havida na continuidade do trabalho realizado nas várias regiōes do Estado (Tabela 2), devido a fatôres orçamentários, em parte superados pela introdução do método seletivo.

Por outro lado, os inquéritos sorológicos em andamento, pela coleta de amos- 
ROCHA e SILVA, E. O. da; DIAS JR., J. \& GUARITA, O. F. - Suspensão do rocia to no combate ao Triatoma infestans. Rev. Saúde públ., S. Paulo, 3(2):173-181, dez. 1969.

T A B E L A 2

Areas trabalhadas pelo SEMPDC - 1968

Indices gerais de infestação das casas

tndices gerais de infecção dos triatomineos

\begin{tabular}{|c|c|c|c|c|c|c|c|}
\hline \multirow[b]{2}{*}{ fndices gerais de } & \multicolumn{7}{|c|}{ Regiăo Administrativa } \\
\hline & $\begin{array}{c}4 \\
\text { Soro- } \\
\text { caba }\end{array}$ & $\begin{array}{c}\mathbf{5} \\
\text { Campl- } \\
\text { nas }\end{array}$ & $\begin{array}{c}6 \\
\text { Rib. } \\
\text { Prêto }\end{array}$ & $\begin{array}{c}7 \\
\text { Bauru }\end{array}$ & $\begin{array}{c}8 \\
\text { S. J. Rlo } \\
\text { Prêto }\end{array}$ & $\begin{array}{c}9 \\
\text { Araca- } \\
\text { tuba }\end{array}$ & $\begin{array}{c}10 \\
\text { Pres. } \\
\text { Pru- } \\
\text { dente }\end{array}$ \\
\hline Infestação & 5,2 & 0,2 & 3,7 & 1,2 & 3,4 & 1,2 & 0,5 \\
\hline Infecção & 7,6 & 0,0 & 1,2 & 4,9 & $\mathbf{1 , 5}$ & 2,4 & 0,4 \\
\hline
\end{tabular}

tras nas escolas primárias do Estado (grupo etário de 9 a 14 anos) revelaram até agora, em 118 municípios terminados, um índice de infecção de $1,19 \%$ em 10.058 amostras examinadas (120 soros reagen. tes).

Atualmente, encontra-se o SEMPDC em condiçōes de substituir a pesquisa anual de casa em casa, em muitos municipios outrora infestados pelo $T$. infestans, por outros métodos de trabalho. Nesses municípios atingiu-se a etapa final da cam. panha de contrôle da espécie domiciliária, ou seja, a fase de Vigilância em Chagas, formulada por DiAs ${ }^{\mathfrak{5}}$ (1956).

No Estado de São Paulo, a Vigilância permitirá a participação ativa da população através da notificação de focos (re- siduais ou novos) além da realização de amplos levantamentos imunológicos, capazes de confirmar a ausência da transmissão natural da doença na área, e ain$\mathrm{da}$, a concentração de esforços visando melhorar os conhecimentos sôbre espécies como o $T$. sordida e o $P$. megistus, uma vez que os exemplares dessas espécies não estão reagindo da mesma forma que o $T$. infestans aos métodos de combate atualmente utilizados, como se depreende de sua aproximação cada vez mais freqüente da moradia, embora sem maiores alterações nos indices de infecção (Tabelas 3 e 4 e Figura 1). Essas atividades serão possiveis pela liberação de recursos decorrentes da suspensão da pesquisa anual de casa em casa nos municípios referidos.

T A B E L A 3

Movimento geral $-T$. sordida

Período: 1951-1968

\begin{tabular}{|c|c|c|c|c|}
\hline \multirow{2}{*}{ Periodo } & \multicolumn{3}{|c|}{ Número de Exemplares } & \multirow{2}{*}{$\begin{array}{c}\% \text { de } \\
\text { infeçãa }\end{array}$} \\
\hline & capturados & examinados & infectados & \\
\hline 1951 a 1955 & 4.116 & 2.894 & 104 & $\mathbf{3 , 5 9}$ \\
\hline 1956 a 1960 & 42.657 & 30.430 & 126 & 0,41 \\
\hline 1961 a 1965 & 168.955 & 43.354 & 717 & 1,65 \\
\hline 1966 a 1968 & 222.078 & 123.931 & 1.582 & 1,26 \\
\hline
\end{tabular}


ROCHA e SILVA, E. O. da; DIAS JR., J. \& GUARITA, O. F. - Suspensão do rocia to no combate ao Triatoma infestans. Rev. Saude públ., S. Paulo, 3(2):173-181, dez. 1969.

\begin{tabular}{|c|c|c|c|c|}
\hline \multirow{3}{*}{ Periodo } & $\begin{array}{r}\mathbf{T} \\
\text { Iovimento } \\
\text { Peri }\end{array}$ & $\begin{array}{c}\text { E L A } 4 \\
\text { P. megistu } \\
1951-1968\end{array}$ & & \multirow{3}{*}{$\begin{array}{c}\% \text { de } \\
\text { infecção }\end{array}$} \\
\hline & \multicolumn{3}{|c|}{ Número de Exemplares } & \\
\hline & capturados & examinados & infectados & \\
\hline 1951 a 1955 & 2.539 & 375 & 1 & 0,26 \\
\hline 1956 a 1960 & 3.735 & 2.532 & 168 & 6,63 \\
\hline 1961 a 1965 & 11.571 & 5.947 & 127 & 2,31 \\
\hline 1966 a 1968 & 21.410 & 14.829 & 357 & 2,40 \\
\hline
\end{tabular}

Desde que continue caindo a densidade e o número dos focos domiciliares e peridomiciliares do $T$. infestans os proble. mas para o futuro, no Estado de São Paulo, serão: realização de operações de ataque com caráter prioritário nas áreas residuais; manutenção de operações de Vigilância nas demais áreas; esclarecimento do significado dos focos silvestres do $T$. infestans na infestação das casas; determinação da importância das espécies semidomésticas ( $T$. sordida e $P$. megistus) na transmissão da doença de Chagas ao homem; aprimoramento dos métodos de combate às espécies semidomésticas; desenvolvimento de campanha visando a prevenção da transmissão da doença de Chagas, pela transfusão de sangue.

\section{FASES DE UMA CAMPANHA}

Tomando como ponto de referência o pressuposto de que existem analogias entre as campanhas de erradicação da malária e aquelas que visam o contrôle dos vetores domiciliários da doença de Chagas, podemos considerar que esta última apresenta distintas etapas no seu desenvolvimento. Em linhas gerais, essas etapas, ou fases seriam as seguintes (Figura 2):

I. Fase Preparatória - na qual se desenvolvem, entre outras, as seguintes atividades:
Levantamento da área endêmica (infecção e doença) ;

levantamento das espécies vetoras (conhecimento, índice de infecção e hábitos) ;

levantamento de recursos e programação de atividades;

seleção e adestramento de pessoal;

reconhecimento geográfico e levantamento sócio-econômico da área;

início da divulgação da campanha.

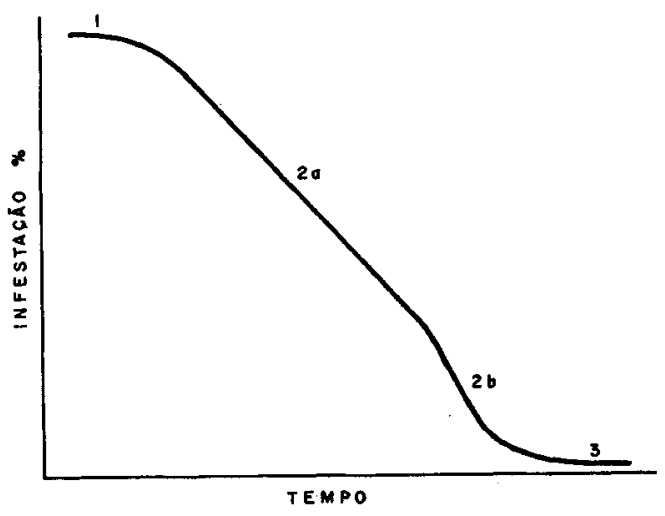

\footnotetext{
1 - FASE PREPARATÓRIA

2 - FASE DE ATAQUE - 0 : iniciol tarrostoo-desinsetizacdo de todas os cosos e onexos ) b: final (seletivo con revisāo de tocos) 3 - FASE DE VIGILÂNCIA
}

Fig. 2 - Evolução hipotética de uma campanha de contrôle do vetor domiciliário $e$ peridomiciliario da doenca de Chagas. 
ROCHA e SILVA, E. O. da; DIAS JR., J. \& GUARITA, O. F. - Suspensão do rociato no combate ao Triatoma infestans. Rev. Saúde públ., S. Paulo, 3(2):173-181, dez. 1969.

II. Fase de Ataque - a) ataque inicial: rociado periódico da área infestada por triatomíneos vetores, pelo método de arrastão, ou seja, expurgo de tôdas as casas e anexos. Sendo possivel, realizar concomitantemente uma pesquisa de triatomíneos, visando a avaliação periódica dos resultados alcançados pela desinsetização. A partir do momento em que a redução dos focos existentes atinja tal número que seja mais econômica a sua procura de casa em casa, através de uma pesquisa razoàvelmente bem feita, atin. giremos a sub-fase seguinte; b) final de ataque: pesquisa periódica de triatomíneos de casa em casa (inclusive anexos) pelo método manual com lanterna e desalojante; revisão dos focos expurgados, tantas vêzes quanto necessário, visando sua eliminação (seguimento e investigação de focos).

De acôrdo com as condições do trabalho realizado e, naturalmente, em função da situação epidemiológica vigente em cada área ao fim de um período de tempo variável, passa-se à fase seguinte.

III. Fase de Vigilância - A pesquisa periódica e a desinsetização seletiva, com a sistemática revisão dos focos, deverá levar ao encontro de um número cada vez menor de exemplares de $T$. infestans, desde que tal espécie seja, realmente domiciliária e semidomiciliária, na área considerada. Sendo assim, para uma área passar da fase de ataque para a de Vigilância, será necessário que os trabalhos levados a efeito na mesma tenham atin. gido determinados resultados.

No Estado de São Paulo, os resultados alcançados são avaliados por uma comissão de técnicos, os quais encaminham um relatório à Diretoria do SEMPDC, justificando a viabilidade ou não da passagem de cada município estudado para a fase de Vigilância,

Os critérios que, no momento, servem de base para a avaliação dêsses resultados, foram aprovados em reunião, da qual participaram todos os técnicos em atividade no SEMPDC. Embora não representando ainda critérios ideais, êles parecem válidos, principalmente se levarmos em conta a inexistência de experiência anterior mais ampla, nesse campo. Os critérios aprovados foram os seguin. tes:

a) Nas avaliações serão levados em consideração, de acôrdo com a oportuni. dade e as condiçôes locais, fatôres de natureza:

\section{Epidemiológica ;}

Ecológica ;

Sócio-econômica ;

Operacional,

Administrativa.

b) As espécies de triatomíneos existentes no Estado de São Paulo, para efeito dessas avaliações, serão considerados como:

Espécie doméstica e semidoméstica: $T$. infestans;

Espécies semidomésticas e silvestres: T. sordida e P. megistus;

Espécies silvestres: as demais.

c) A unidade de estudo para efeito de avaliação será o município. Em casos especiais, a critério do Grupo de Trabalho, poderá ser avaliado o município que apresente área parcial contínua, que atenda aos presentes critérios e que abran. ja pelo menos $50 \%$ das localidades e das casas existentes, excluída a zona urbana. Nessa área parcial contínua poderá ser suspenso o rociado seletivo e nela serão desenvolvidas atividades próprias $\mathrm{da} \mathrm{Vi}$ gilância.

d) Não poderá ser considerado em condiçóes de entrar em Vigilância município em que, nos dois últimos anos, foram capturados exemplares de $T$. infes. tans infectados por tripanossomas do tipo cruzi. 
ROCHA e SILVA, E. O. da; DIAS JR., J. GUARITA, O. F. - Suspensáo do roclàto no combate ao Triatoma infestans.

Rev. Saude publ., S. Paulo, 3(2):173-181, dez. 1969.

e) Sòmente poderá ser considerado em condiçóes de ser avaliado, o município que tenha sido trabalhado no ano anterior, pelo método seletivo, com pesquisas em pelo menos $95 \%$ das casas, excluída a área urbana.

f) Para conhecer a proteção dada pelo SEMPDC ao municipio em estudo, será realizado um levantamento das desinsetizaçôes anteriores visando esclarecer, entre outros aspectos, a periodicidade do trabalho, sua expansão e métodos empregados.

g) Será feito um estudo comparativo dos indices gerais anuais de infestação e infecção por espécie, em casas e anexos, nos três últimos anos.

h) Não poderá passar para a fase de Vigilância, município que apresente mais de $5 \%$ das suas localidades infestadas, em pesquisa realizada no ano anterior $e$ ainda:

- para pesquisas intradomiciliares, em qualquer dessas localidades, mais de $5 \%$ de positividade;

- para pesquisas peridomiciliares, em qualquer dessas localidades, mais de $10 \%$ de positividade.

i) $\mathrm{Na}$ avaliação de um município, será levada em conta também a situação epidemiológica dos municípios vizinhos, especialmente das localidades limítrofes.

j) Quando presentes, deverāo ser avaliados fatôres e condiçôes de processamento de migração e do trânsito de pessoas e animais pelo município (infestação passiva).

k) A fim de estabelecer a receptividade do município, deverão ser levantados dados sôbre os índices de casas de barro e pau-a-pique, por localidade e estabelecer sua relação com os índices de infestação no três últimos anos. Pelo mesmo motivo, quando houver, deverão ser estudados os levantamentos sorológicos realizados.
1) É conveniente que existam estudos de investigação de focos anteriores à avaliação, sabendo-se que, na Vigilância, essa prática será obrigatória para esclarecimento das condiçóes, da natureza, da extensão e, se possível, dos focos residuais ou novos. Para alcançar plenamente seus objetivos, a investigação não poderá ficar restrita à casa e anexos.

m) 0 conhecimento da distribuição das unidades escolares terá valor, assim como, os meios e facilidades de comunicação entre a zona rural e a sede municipal, principalmente se relacionados aos fatôres epidemiológicos mais importantes, presentes no município.

n) Em separado, será realizada a avaliação da área periurbana da sede municipal.

\section{METODOS DE TRABALHO PARA AREAS EM VIGILANCIA}

As atividades de contrôle recomendadas para áreas em Vigilância, são as seguintes:

a) Instalação de uma rêde de Postos de Informação de Focos de Triatomíneos (PIFT) (Rocha e Silva; Lavorini \& SCORNAIENCHI ${ }^{13}$ ).

b) Garantia do funcionamento da rêde instalada, através não só da supervisão e estímulo, mas também pelo atendimento imediato das notificações recebidas.

c) Investigação dos focos detectados e seu posterior seguimento.

d) Caixas-abrigo coletoras de "harbeiros" (método Gómez-Núñez) (ForatTini, JUAREZ \& CORRÊA ${ }^{7}, 1969$ ).

e) Levantamentos imunológicos realizados em diversos grupos etários.

f) Nos focos detectados, xenodiagnóstico de animais. 
ROCHA e SILVA, E. O. da; DIAS JR., I. \& GUARITA, O. F. - Suspensão do rociato no combate ao Triatoma infestans. Rev. Saúde públ., S. Paulo, 3(2):173-181, dez. 1969.

g) Divulgação sanitária, visando esclarecer aspectos da doença de Chagas, principalmente os relacionados com o modo de transmissão, hábitos do vetor e seu combate. Será realçada também a importância da notificação dos focos de triatomíneos, o papel da limpeza da casa $e$ afastamento dos anexos, no contrôle dos vetores.

h) Pesquisa cuidadosa de tôdas as casas e anexos, realizada por pessoal especialmente adestrado para trabalhar nessas áreas. Essa pesquisa deverá ser realizada de três em três anos, na zona rural e periurbana e servirá como elemento de avaliação dos resultados alcançados. A aplicação dos métodos acima preconizados, exceção feita ao último, não obedecem uma rotina pré-estabelecida. Serão aplicados em função da situação epidemiológica de cada área em Vigilância.

\section{AREAS EM VIGILÂNCIA}

No início de 1969 foram realizadas no Estado de São Paulo, as primeiras avaliações epidemiológicas visando a passagem de alguns municípios da Região 7 (Bauru) e Região 10 (Presidente Prudente) para a fase de Vigilância.

Nessa ocasião, foram escolhidos para servirem de área pilôto em Vigilância, os municípios da Região de Bauru: Pirajuí, Guarantã, Presidente Alves, Pongaí, Balbinos e Uru. Abrangem êles uma área de $61.867 \mathrm{~km}^{2}$ e apresentam uma população estimada em 51.000 habitantes. Nessa área pilôto já estão sendo aplicados e avaliados os métodos selecionados.

Recentemente, foram realizados cursos de adestramento para divulgadores, chefes de setor e capturadores, no sentido de preparar o pessoal que deverá atuar nessas áreas.

Relatamos, ao finalizar, a boa receptividade obtida junto à população, em particular o professorado, pelas atividades de notificação de focos de triatomíneos.
Rocha e Silva, E. O. da; Dias Júnior, J. \& GuARITA, O. F. - Suspension of spraying in the combat of Triatoma infestans in certain areas of the State of São Paulo, Brazil. Rev. Saúde puibl., S. Paulo, 3(2):173-181, dez. 1969.

Summary - The authors present at the beginning, the evolution of the attack against $T$. infestans, main vector of Chagas' Disease in the State of São Paulo (Brazil). They stress that at first there was a decline in the density of these insects in the counties worked periodically. This decline in density was followed by the reduction of the infection rate, by $T$. cruzi and Machado Guerreiro positive reactions. When the conditions became suitable, Pedreira de Freitas' Selective method was introduced, making possible the improvement of the spraying technique and capture as well as the amplification of the protected area. They consider that a control campaing of the domiciliary vectors of Chagas' Disease present different phases and they try to explain when and how, in some counties in the State of São Paulo they reached the Surveillance phase as preconized by Emmanuel Dias. A pilot area was established, where methods of work are being studied and people hired in the Surveillance areas are being trained. Finally, they show that $T$. sordida and $P$. megistus are not reacting satisfactorily to the present method of attack.

\section{A G R D E C I M E N T O S}

Aos funcionários do SEMPDC que direta ou indiretamente colaboraram na elaboração dêste trabalho. Aos técnicos: José Maluf, George K. Ishiata e Argemiro Fomm, pelas sugestōes e entusiasmo com que aceitaram as idéias aqui explanadas.

\section{REFERENCIAS BIBLIOGRAFICAS}

1. CORREA, R. R. \& FERREIRA, O. A. - Distribuição geográfica, habitats $e$ infecção do Triatoma sordida no Estado de São Paulo, Rev. Inst. Med. trop. S. Paulo, 1:207-213, set./out. 1959. 
ROCHA e SILVA, E. O. da; DIAS JR., J. \& GUARITA, O. F. - Suspensão do rocia to no combate ao Triatoma infestans. Rev. Saúde públ., S. Paulo, 3(2):173-181, dez. 1969.

2. CORREA, R. R.; FOMM, A. S. \& DIAS JR., J. - Estudo comparativo de métodos de captura de triatomineos transmissores da doença de Chagas. Rev. paul. Med., 71:280-297, dez. 1967.

3. CORREA, R. R.; LIMA, F. O. \& CARVALHO, P. J. - Da infecção natural pelo Trypanosoma cruzi nas ninfas e adultos do Triatoma infestans Arq. Hig., S. Paulo, 17:9-14, mar. 1952

4. COUTINHO, J. O. - Contribuicão ao estudo da epidemiologia do doenca de Chagas. Arq. Hig., S. Paulo, 27:317-330, dez. 1962 .

5. DIAS, E. - Profilaxia da doenca de Chagas. [Conferência realizada na Academia Nacional de Medicina]. Jornal do Comércio, Río de Janeiro, 2-12-1956.

6. DIAS, E. \& PELLEGRINO, J. - Alguns ensaios com o gamexane no combate aos transmissores da doença de Chagas. Brasil-méd., 62:185-191, maio 1948.

7. FORATTINI, O. P.; JUAREZ, E. \& CORREA, R. R. - Medida da infestacão domiciliar por Triatoma infestans. Rev. Saúde públ., S. Paulo, 3:11-16, jun. 1969.

8. LIMA, F. O. \& SILVA, T. L. da Distribuição dos triatomíneos no Estado São Paulo. Arq. Hig., S. Paulo, 17:27-55 , mar. 1952.

9. PAUlini, E.; FOMM, A. S. \& GUEDES, A. S. - Ensaio sôbre desalojantes no combate à doença de Chagas. Rev. bras. Malar., 13:64-69, jan./ jun. 1961.
10. PEDREIRA de FREITAS, J. L. - Importância do expurgo seletivo dos domicilios e anexos para a profilaxia da moléstia de Chagas pelo combate aos Triatomineos. Arq. Hig., S. Paulo, 28: 217-272, set. 1963 .

11. REIS, U. L. dos; FRANÇA, J. B. M. \& ROCHA e SILVA, E. O. da - Um critério de menor custo como subsídio para a escolha do método de combate a triatomineos vetores da doença de Chagas. Rev. Saúde públ., S. Paulo, 3:31-39, jun. 1969

12. ROCHA e SILVA, E. O. da \& FRANCA, J. B. M. - Profilaxia da doença de Chagas. Sugestōes para uma nova programação de trabalho. Arq. Hig., S. Paulo, 30/31:33-46, 1965/6.

13. ROCHA e SILVA, E. O. da; LAVORINI, M. I. M. \& SCORNAIENCHI, I. M. - Instalação de uma rêde de postos de informação de focos de triatomineos (PIFT). Arq. Hig., S. Paulo (em via de publicação).

14. SCHIAVI, A.; LIMA, A. R. \& RAMOS, A. S. - A desinsetização da área central do Estado de São Paulo, visando os vetores da moléstia de Chagas. Arq. Hig., S. Paulo, 17:117-121, mar. 1952.

15. UNTI, O. \& SILVA, T. L. da - Levantamento da moléstia de Chagas no Estado de São Paulo pela reação sorológica. Arq. Hig., S. Paulo, 17:123-132, mar. 1952 . 